\title{
To the morphological heterochromatin condensation state in granulocytic progenitors - myeloblasts - in patients suffering from the myelodysplastic syndrome and acute myeloblastic leukemia
}

\author{
Smetana K*, Klamová H, Mikulenková D and Čermák J \\ Institute of Hematology and Blood Transfusion, Prague, Czech Republic
}

\begin{abstract}
Background: According to previous study the heterochromatin condensation state (HChCS) of myeloblasts in acute myeloblastic leukemia M2 type (M2 AML) with the altered differentiation exhibited a remarkable similarity in both nuclear central and peripheral regions. In contrary, HChCS was larger in central nuclear regions of myeloblasts with the differentiation potential in patients suffering from the chronic phase of the chronic myeloid leukemia (CML). The present study was undertaken to provide more information whether at least some myeloblasts in refractory anemia (RA) without or with excess of myeloblasts (EB) of the myeloblastic syndrome (MDS) might resemble those in AML.
\end{abstract}

Methods: The HChCS in myeloblasts and mature granulocytes was studied in bone marrow smears of 3 patients with chronic phase of Ph1+ chronic myeloid leukemia (CML), 3 patients with RA MDS (myeloblasts <5\%), 3 patients with EB MDS (myeloblasts >9\%) and 3 patients with M2 AML (myeloblasts > 20\%). The heterochromatin optical density reflecting its condensation state ( $\mathrm{HChCS}$ ) was measured in 3 central and 3 peripheral nuclear regions of each measured nucleus. The similarity or difference of the central and peripheral heterochromatin density was expressed by the ratio of the mean value of the central to peripheral heterochromatin density calculated for each measured cell.

Results: The heterochromatin optical density image measurements at "single cell level" demonstrated that some myeloblasts with similar HChCS in nuclear and peripheral nuclear regions in AML are present already in patients suffering from the MDS. However, the incidence of these cells in MDS was naturally smaller than in AML. The larger HChCS in central nuclear regions of myeloblasts of MDS or AML patients was similar to most of myeloblasts with the differentiation potential in CML.

Conclusion: Some myeloblasts in MDS exhibited a remarkable similarity of the HChCS in both central and peripheral nuclear regions. Such myeloblasts resembled myeloblasts with the altered differentiation in M2 AML. Myeloblasts with the larger HChCS in the central nuclear region in MDS and AML resembled myeloblasts of CML. with the differentiation potential. Such myeloblasts might be responsible for the incidence of mature granulocytes in the peripheral blood of MDS and AML patients.

\section{Introduction}

In the hematological cytology the heterochromatin morphology is a very useful tool for the cell identification including the differentiation and maturation stage. It should be also mentioned that heterochromatin represents nuclear regions which reflect sites of silent genes [1,2]. The heterochromatin heavy condensation state and structural organization prevent the gene activation and insure the genome stability [3-5].

Previous studies on myeloblasts of chronic myeloid leukemia (CML) demonstrated different heterochromatin condensation state (HChCS) in the "central gene rich" and "peripheral gene poor" nuclear regions [6]. On the contrary, in acute myeloblastic leukemia (M2 AML) some myeloblasts did not exhibit such difference of HChCS in central and peripheral nuclear regions. Such similarity of HChCS in central and peripheral nuclear regions was also observed in fully differentiated and mature segmented granulocytes. Thus it appeared likely that some M2AML myeloblasts exhibited a premature terminal differentiation that might be related to the known altered differentiation process of leukemic progenitors [7-9].
The present study was undertaken to provide more information whether myeloblasts with similar HChCS in central and peripheral nuclear regions are present already in patients suffering from the refractory anemia (RA) of the myelodysplastic syndrome (MDS) that may precede the acute myeloid leukemia [10-13]. At this occasion it should be noted that the term myeloblasts in the present study was used for "agranular myeloblasts" mentioned in previous studies on MDS [14]. The results of the heterochromatin optical density image measurements demonstrated that such myeloblasts were present already in RA MDS without or with excess of myeloblasts (EB). However, the

${ }^{*}$ Correspondence to: Karel Smetana, Institute of Hematology and Blood Transfusion, U nemocnice 1, Prague, 128 20, Czech Republic, E-mail: karel. smetana@uhkt.cz

Key words: Heterochromatin, myeloblasts, myelodysplastic syndrome, acute myeloblastic leukemia

Received: January 16, 2020; Accepted: January 23, 2020; Published: January 27, 2020 
Smetana K (2020) To the morphological heterochromatin condensation state in granulocytic progenitors - myeloblasts - in patients suffering from the myelodysplastic syndrome and acute myeloblastic leukemia

incidence of these cells was naturally smaller than in M2 AML. At this occasion it should be mentioned that the similarity of HChCS in both central and peripheral nuclear regions was characteristic for terminally differentiated mature granulocytes in all studied patients suffering from RA MDS, EB MDS and M2 AML. In addition, terminally differentiated mature granulocytes of CML patients or non-leukemic persons also exhibited such similarity as described previously [6].

\section{Material and methods}

The HChCS in myeloblasts and mature granulocytes were studied in bone marrow smears of 3 patients with chronic phase of $\mathrm{Ph} 1+$ CML, RA MDS (myeloblasts $<5 \%$ ), EB MDS (myeloblasts $>9 \%$ ) and M2 AML (myeloblasts >20). All these hematological disorders were classified according to common characteristic clinical and laboratory markers including the cytology, genetics and FACS phenotyping. For comparison, the HChCS was also studied in peripheral blood smears of differentiated and mature granulocytes of 3 non-leukemic persons blood donors. The bone marrow biopsies and peripheral blood smears were originally taken for diagnostic purposes and approved protocols by the Institute authorities.

Bone marrow and peripheral blood smears were stained by the May-Grünwald - Giemsa-Romanowsky (MGGR) standard polychrome staining procedure. This procedure is useful for both identification of bone marrow cells and also for the chromatin visualization as a histochemical tool including heterochromatin optical density measurements $[6,15]$.

Micrographs were captured with a Camedia digital camera C4040 ZOOM (Olympus, Japan) placed on Jenalumar microscope (Zeiss, Germany). The double adapter on the microscope increased the magnification of captured images transferred to the computer screen and facilitated density measurements at the single cell level.

The heterochromatin image optical density was measured after the conversion of captured color signals to gray scale using the red channel of NIH Image Program - Scion for Windows (Scion Corp., USA). The heterochromatin optical density reflecting its condensation state was measured in 3 central and 3 peripheral nuclear regions of each measured nucleus and was expressed in arbitrary density units (Figure 1 and 2). These arbitrary density units (AU) were calculated by subtracting the mean background density surrounding the measured cell from the mean heterochromatin density in central or peripheral nuclear regions [6]. The similarity or difference of the central and peripheral heterochromatin density was expressed by the ratio of the mean value of the central to peripheral heterochromatin density calculated for each measured cell [9]. Such calculation facilitated the comparison of results achieved by image optical density measurements of single cells in smear preparations with different bone marrow or peripheral blood smearing. The variation coefficient of the background density surrounding each cell in different bone marrow smears was $17.6 \pm 1.9$ per cent. The results of all measurements at the single cell level such as mean, standard deviation significance and variation coefficient were evaluated using Primer of Biostatistic Program, version 1 developed by S.A. Glantz (McGraw-Hill, Canada, 1968).

\section{Results}

\section{CML}

As it was reported previously [6,15], most of myeloblasts in patients suffering from the chronic phase of CML exhibited the heavier HChCS in central nuclear regions in comparison with the nuclear periphery (Table 1). During the further differentiation and maturation of granulocytic lineage such difference disappeared. HChCS in central and peripheral nuclear regions of differentiated cells - segmented granulocytes appeared to be very similar. Thus the central to peripheral HChCS ratio was smaller in these cells than in myeloblasts $[6,16]$ (Table 1).

\section{RA MDS and EB MDS}

The incidence of myeloblasts with a larger HChCS in nuclear central regions in both RAMDS and EBMDS was markedly smaller in comparison with CML (Table 1). Slightly above fifty per cent of myeloblasts exhibited similar HChCS in both central and peripheral nuclear regions. Thus the central to peripheral HChCS ratio in these was reduced (Table 1 and Figure 1). The HChCS in nuclear and peripheral regions of differentiated and mature cells of the granulocytic cell lineage such as segmented granulocytes in these patients also exhibited a marked similarity reflected by the decreased central to peripheral HChCS ratio (Table 1). On the hand, less than $50 \%$ of myeloblasts exhibited a larger HChCS in nuclear central regions and resembled those in CML (Figure 2).

\section{M2AML}

The HChCS in myeloblasts of patients suffering from M2AML was mostly similar in both central and peripheral nuclear regions [9] (Table 1). Such similarity of HChCS in central and peripheral regions was also noted almost in all differentiated and mature neutrophils with segmented nuclei. Thus the central to peripheral HChCS ratio in all these cells was small. On the other hand, a small number of myeloblasts exhibited a heavier HChCS in the nuclear central region than in the nuclear periphery (Table 1).

\section{Non-leukemic persons}

The HChCS of bone marrow myeloblasts was not studied in nonleukemic persons. In these patients the number of myeloblasts was not satisfactory for measurements and in blood donors it was not justifiable to perform bone marrow biopsies. Nevertheless, HChCS was similar in central and peripheral nuclear regions of differentiated

Table 1. The central/peripheral HChCS expressed in arbitrary optical density units in myeloblasts and mature differentiated neutrophylic granulocytes of CML, RA MDS. EB MDS, M2 AML patients and non-leukemic patients*

\begin{tabular}{|c|c|c|c|}
\hline Patients & $\begin{array}{l}\mathrm{Ctr} / \mathrm{Prph} \mathrm{HChCS} \\
\text { Mean }\end{array}$ & $\begin{array}{l}\mathrm{Ctr} / \mathrm{Prph} \mathrm{HChCS} \\
\text { with mean }<1.1\end{array}$ & $\begin{array}{l}\text { Percentage of cells } \\
\text { with mean }<1.1\end{array}$ \\
\hline \multicolumn{4}{|c|}{ Myeloblasts } \\
\hline$C M L$ & $1.23 \pm 0.09$ & $0.97 \pm 0.05$ & $9.0 \pm 0.3^{\# \#}$ \\
\hline RA MDS & $1.11 \pm 0.31$ & $1.01 \pm 0.04$ & $57.5 \pm 0.4^{\#}$ \\
\hline EB MDS & $1.12 \pm 0.26$ & $0.97 \pm 0.05$ & $59.0 \pm 0.5^{\#}$ \\
\hline M2 AML & $1.03 \pm 0.06$ & $0.97 \pm 0.06$ & $76.4 \pm 0,4$ \\
\hline \multicolumn{4}{|c|}{ Mature Segmented Neutrophils } \\
\hline$N L P$ & $1.01 \pm 0.01$ & ----- & $\sim 98$ \\
\hline$C M L$ & $0.93 \pm 0.20$ & ------ & $\sim 99$ \\
\hline RA MDS & $1.00 \pm 0.07$ & ----- & $\sim 98$ \\
\hline EB MDS & $0.93 \pm 0.02$ & ----- & $\sim 99$ \\
\hline M2 AML & $1.02 \pm 0.11$ & ------ & $\sim 99$ \\
\hline
\end{tabular}

* - Mean and standard deviation based on $\sim 100$ measurements in myeloblasts of 3 RAMDS patients, $\sim 200$ measurements in myeloblasts of 3 EBMDS patients, $\sim 200$ measurements in myeloblasts of $3 \mathrm{M} 2 \mathrm{AML}$ patients, 100 measurements in myeloblasts of $3 \mathrm{CML}$ patients, more than 100 mature segmented neutrophils of each group of RA MDS, EB MDS, M2 AML, CML and NLP (non-leukemic patients).

\#\# - Significant difference from RA MDS, EB MDS, M2 AML using t-test ( $(2 \alpha=0.05)$. \#- Significant difference from M2 AML using t-test $((2 \alpha=0.05)$.

$\mathrm{Ctr} / \operatorname{PrphHChCS}$ - heterochromatin condensation state in central to peripheral nuclear region ratio. 
Smetana K (2020) To the morphological heterochromatin condensation state in granulocytic progenitors - myeloblasts - in patients suffering from the myelodysplastic syndrome and acute myeloblastic leukemia

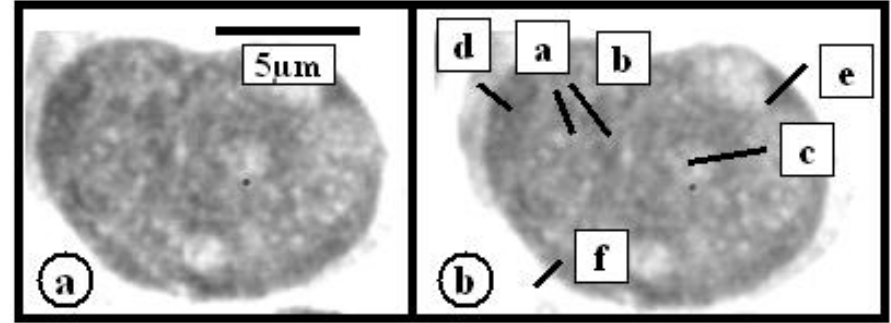

Figure 1. RA MDS myeloblast (a). (b) Density measurement lines - black bars in central a,b,c - and peripheral - d,e,f nuclear regions. Ctr/Prph HChCS calculated ratio is following $[(\mathrm{a}+\mathrm{b}+\mathrm{c}): 3=70.6 \mathrm{AU}]:[(\mathrm{d}+\mathrm{e}+\mathrm{f}): 3=71.4 \mathrm{AU}]=0.98$

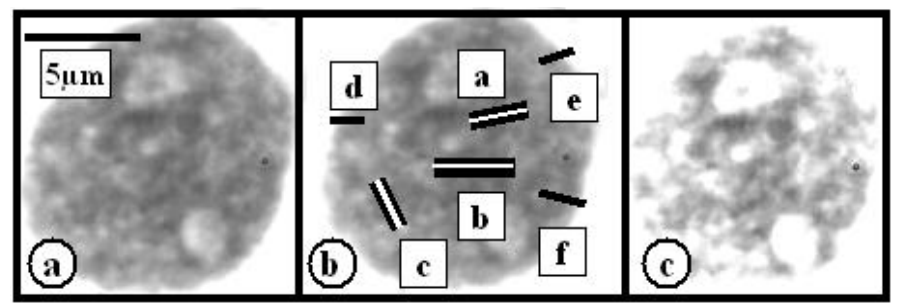

Figure 2. RA MDS myeloblast (a). (b) Density measurement lines - black bars in centra - a,b,c - and peripheral - d,e,f - nuclear regions. Ctr/Prph HChCS calculated ratio is following $[(\mathrm{a}+\mathrm{b}+\mathrm{c}): 3=74.5 \mathrm{AU}]:[(\mathrm{d}+\mathrm{e}+\mathrm{f}): 3=59.0 \mathrm{AU}]=1.26$. After computer processing such as bleaching and contrast increasing the heterochromatin in central nuclear regions exhibits a larger density than in the nuclear periphery

mature granulocytes with characteristic nuclear segmentation (Table 1). The satisfactory number of these cells for density measurement was naturally easily accessible in diagnostic peripheral blood smears of non-leukemic patients or blood donors.

\section{Discussion}

It seems to be likely that some myeloblasts of RA without and with EB in patients suffering from MDS exhibited a remarkable similarity to those in M2 AML. Such similarity was due to the similar HChCS in both central and peripheral nuclear regions. In myeloblasts of M2 AML this similarity apparently reflected a premature differentiation of these cells related to the altered differentiation [9]. Such observation seems to be in harmony with the presumption that in some patients MDS may precede the AML including the M2 AML [10-14,17]. However, it would be difficult to express whether these cells in MDS represent already leukemic progenitor cells. The morphological methods are limited to solve such supposition that would require more sophisticated approach $[13,18]$. At this occasion it should be noted that in fully differentiated and mature granulocytes of patients with CML or non-leukemic persons there was not any distinct difference of HChCS between nuclear central and peripheral regions similarly as in some myeloblasts of MDS and most myeloblasts of M2 AML.

The larger HChCS in central nuclear regions in a small number of myeloblasts in MDS (Figure 2) or M2 AML patients was similar to most of myeloblasts with the differentiation potential in CML: Such myeloblasts in MDS and M2 AML might reflect their ability to differentiate and might be responsible for the presence of differentiated mature granulocytes in the peripheral blood of these patients. The incidence of 2 different populations with of myeloblasts with different proliferation activity or morphology in patients with acute myeloblastic leukemia was already reported and discussed previously. However, these reports were based on different methodical approaches $[19,20]$.

At the end of the discussion it must be noted that the goal of the present study was to show whether already some myeloblasts in RA and EB MDS exhibited a similarity of HChCS to those in M2 AML. The relationship of the incidence of these myeloblasts to the clinical state of studied patients in MDS and M2AML was not a target of the present study because of the small number of studied patients. Nevertheless, the present study might provide a complementary morphological information to more sophisticated studies on the MDS development and transition to acute myeloid leukemias and especially to M2 AML [18].

\section{Acknowledgement}

The study was partially supported by the Research Program of the Institute of Hematology and Blood Transfusion. The authors would like to express their gratitude to physicians and technicians of the Institute for the continuous support.

\section{References}

1. Grigoryev SA, Bulynko YA, Popova EY (2006) The end adjust the means: heterochromatin remodeling during terminal cell differentiation. Chromosome Res 14: 53-69.

2. Politz JCR, Scalzo D, Groudine W (2013) Something silent this way forms: the functional organization of the regressive nuclear compartment. Annu Rev Cell Dev Biol 29: 101512-122317.

3. Alcobia I, Dilao R, Parreira L (2000) Spatial associations of centromeres in the nucle of hematopoietic cells: evidence for cell-type-specific organizational patterns. Blood 95:1608-1615.

4. Cremer T, Cremer C (2005) Rise, fall and resurrection of chromosome territories: a historical perspective. Part II. Fall and resurrection of chromosome territories during 1950s to 1980. Part III. Chromosome territories and the functional nuclear architecture: experiments and models from 1990s to the present. Europ J Histochem 50: 223-372.

5. Cohen AL, Jia S (2014) Noncoding RNAs and the borders of heterochromatin. Wiley Interdiscip Rev RNA 5: 835-847. [Crossref]

6. Smetana K, Mikulenková D, Klamová H (2011) Heterochromatin density (condensation) during cell differentiation and maturation using the human granulocytic lineage of chronic myeloid leukaemia as a convenient model. Folia Biol (Praha) 57: 216-211

7. Gavosto F, Pileri A, Bachi C, Pegoraro L (1964). Proliferation and maturation defect in acute leukaemia cells. Nature 203:92-94.

8. Cáceres-Cortés JR (2013) Blastic leukaemias (AML): a biologist's view. Cell Biochem Biophys 66: 13-22. [Crossref]

9. Smetana K, Mikulenkova D, Hrkal Z, Klamova H (2015) On the heterochromatin condensation state diversity in myeloblasts of chronic myelocytic and acute myeloblastic leukemias. Ann Clin Pathol 3: 1056.

10. Shi J, Shao ZH, Liu H, Bai J, Cao YR, et al. (2004) Transformation of myelodysplastic syndromes into acute myeloid leukemias. Chin Med J (Engl) 117: 963-967.

11. Mohammad AA (2018) Myelodysplastic syndrome from theoretical review to clinical application view. Oncol Rev 12: 397

12. Chen J, Kao YR, Sun D, Todorova TI, Reynolds D, et al. (2019) Myelodysplastic syndrome progression to acute myelois leukemia at the stem cell level. Nat Med 26: 103-110.

13. Engelbrecht L, Götze KS, Schwamborn K, Müller-Thomas C, Alpermann T, et al (2019) Correlation of cytomorphology and histopathology in the diagnosic process of myeloid maligancies. Hematol Med Oncol 4: 1-5.

14. Mufti GJ, Bennett JM, Goasguen J, Bain BJ, Baumann I, et al. (2008) Diagnosis and classification of myelodysplastic syndrome (WGM-MDS) consensus proposals for the definition and enumeratio of myeloblasts and ring sideroblasts. Haematologica 93: 1712-1717.

15. Wittekind DH (1983) On the nature of Romanowsky--Giemsa staining and its significance for cytochemistry and histochemistry: an overall view. Histochem $J 15$ : 1029-1047. [Crossref]

16. Smetana K (2014) The heterochromatin condensation state in peripheral acegene poorâ and central â€œgene rich nuclear regions of less differentiated and mature human leukemic cells. A mini-review with additional original observations. J Leuk 2: 1000151. 
Smetana K (2020) To the morphological heterochromatin condensation state in granulocytic progenitors - myeloblasts - in patients suffering from the myelodysplastic syndrome and acute myeloblastic leukemia

17. Ye X, Chen D, Zheng Y, Wu C, Zhu X, et al. (2019) The incidence, risk factors, and survival of acute myeloid leukemia secondary to myeloblastic syndrome: A populationbased study. Hematol Oncol 37: 438-446.

18. Stevens BM, Khan N, D'Alessandro A, Nemkov T, Winters A, et al. (2018) Characterization and targeting of malignant stem cells in patients with advanced myelodysplastic syndromes. Nar Commun 9: 3694
19. Saunders EF, Lampkin BC, Maurer AM (1967) Variation of proliferative activity in leukemic cell populations of patients with acute leukemia. J Clin Invest 48: 1356-1363.

20. McCulloch EA, Minden MD, Buick RN, Izaquirre CA (1978) Clonal expansion and progression in acute myeloblastic leukemia. Bull Cancer 65: 443-448.

Copyright: $(02020$ Smetana K. This is an open-access article distributed under the terms of the Creative Commons Attribution License, which permits unrestricted use, distribution, and reproduction in any medium, provided the original author and source are credited. 